\title{
Screening, diagnosis and genetic study of breast cancer patients in Pakistan
}

\author{
Ayesha Isani Majeed ${ }^{1}$, Asmat Ullah $^{2}$, Muniba Jadoon ${ }^{3}$, \\ Wasim Ahmad ${ }^{4}$, Sheikh Riazuddin ${ }^{5}$
}

\begin{abstract}
Objective: To determine the role of variants in BRCA1 gene in breast cancer development, women of Pakistani origin, diagnosed with breast cancer, were screened for variants in the BRCA1.

Methods: The present study involved screening of 5000 women for breast cancer. 302 women were diagnosed with breast cancer. Using Sanger sequencing, DNA extracted from peripheral blood of 100 patients was screened for disease causing variants in the BRCA1.

Results: Analysis of sequenced data revealed two frame shift (Gly312Trpfs*8, Ala322Glyfs*4), six missense (p.Glu362Lys, p.Lys651Arg, p.Asp693Asn, p.Pro871Leu, p.Glu1134Lys, p.Lys1183Arg), four synonymous (p.Thr327Thr, p.Ser694Ser, p.His771His, p.Gln1135Gln), and two intronic variants (g.75407T>C, g.75401_75401delT) in the patients.

Conclusion: The present investigation showed that variations in BRCA1 made substantial contribution in causing hereditary/early-onset breast cancer in Pakistani women.
\end{abstract}

KEYWORDS: Breast Cancer, Sanger Sequencing, BRCA1 Variants.

doi: https://doi.org/10.12669/pjms.36.2.1059

How to cite this:

Majeed Al, Ullah A, Jadoon M, Ahmad W, Riazuddin S. Screening, diagnosis and genetic study of breast cancer patients in Pakistan. Pak J Med Sci. 2020;36(2):16-20. doi: https://doi.org/10.12669/pjms.36.2.1059

This is an Open Access article distributed under the terms of the Creative Commons Attribution License (http://creativecommons.org/licenses/by/3.0), which permits unrestricted use, distribution, and reproduction in any medium, provided the original work is properly cited.

1. Ayesha Isani Majeed

Department of Radiology,

Pakistan Institute of Medical Sciences, Islamabad, Pakistan.

Health Services Academy, Islamabad, Pakistan.

2. Asmat Ullah,

3. Muniba Jadoon,

4. Wasim Ahmad,

5. Sheikh Riazuddin,

2,3,5: Department of Molecular Biology,

Shaheed Zulfiqar Ali Bhutto Medical University, Islamabad, Pakistan.

2,4: Department of Biochemistry, Faculty of Biological Sciences, Quaid-i-Azam University, Islamabad, Pakistan.

Correspondence:

Wasim Ahmad

Department of Biochemistry,

Faculty of Biological Sciences,

Quaid-i-Azam University,

Islamabad, Pakistan.

Email: wahmad@qau.edu.pk

* Received for Publication:

* $1^{\text {st }}$ Revision Received:

* $\quad 2^{\text {nd }}$ Revision Received:

* Final Revision Accepted:
May 14, 2019

June 17, 2019

October 25, 2019
October 18, 2019

\section{INTRODUCTION}

Breast cancer is designated as one of the major life threatening malignancies throughout the world. As reported by World Health Organization (WHO, 2018), the worldwide incidence is on the rise and attributed to factors like increased life expectancy, increased urbanization and adopted lifestyle. In another report issued by World Cancer Research Foundation, nearly 1.7 million new cases were diagnosed in 2012 worldwide making it second most common cancer overall (www.wcrf.org/int/ cancer-facts-figures/data-specific-cancers/breastcancer-statistics). In Pakistan a comprehensive analysis revealed that breast cancer is the most prevalent, frequently diagnosed and top most common cause of cancer death among the female population. ${ }^{1}$ These authors reported 119,710 cases of breast cancer in the last five years.

In the last few years, several published studies have reported several factors contributing towards high incidence of breast cancer. This included high 
penetrance of inherited cases resulting from genetic mutations. ${ }^{2}$ To date, two major susceptibility genes, $B R C A 1$ and BRCA2, involved in development of breast cancer, have been identified. Genomic rearrangements within these genes are reported to have sound association with breast cancer. ${ }^{3} B R C A 1$ is a tumor suppressor gene, located on chromosome 17q21, and composed of 22 coding and two noncoding exons. ${ }^{4}$ It has a transcript of $7.8 \mathrm{~kb}$ which encodes 1863 amino acids nuclear protein, and play highly pivotal role in transcription and DNA damage repair mechanism. ${ }^{5}$

It has been reported that mutational changes in the BRCA1 confer $80 \%$ life time risk of breast cancer and $40 \%$ of ovarian cancer. ${ }^{6}$ Although, over 500 alterations have been documented for BRCA1, nevertheless the mutational spectrum for this gene has not been characterized entirely. This is due to the fact that these mutational changes revealed varying distribution among different ethnicities and across diverse geographic regions. ${ }^{7,8}$

Pakistan is a geographically dynamic country and home to population with varying ethnicities and geographical linkages. Therefore, there is a need to conduct molecular analyses among the breast cancer patients across different regions of the country and contribute towards defining statistics at the national level. Also, various factors like genetics, life style, obesity, exposure to ionizing radiations have been associated with an increase in cancer. Therefore early detection and breast cancer awareness are the key towards longevity and survival against the disease. ${ }^{9}$ Previously, genetic alterations in the BRCA1 in Pakistani breast cancer patients have been reported only in two different studies. ${ }^{6,10}$ The present study was conducted at the Federal Capital Territory so as to comprehend the mutational changes in BRCA1 among the breast cancer patients of the region.

\section{METHODS}

In total, 5000 females were screened for breast cancer from October 2015 to October 2018 at Federal Breast Cancer Screening Centre, Pakistan Institute of Medical Sciences, Islamabad. The study was approved by Ethical Review Board of Shaheed Zulfiqar Ali Bhutto Medical University, Quaid-i-Azam University Islamabad (Ref. No. IRBQAU-183 dated January 22, 2019). Patients recruited in the study had a diagnosis of primary invasive breast cancer. They were selected irrespective of the patients' age or breast cancer stage at the time of diagnosis. Both the prevalent as well as incident cases were included in the study. Clinical, histopathological and risk factor data such as age, marital status, consanguinity, parity, family history of breast cancer and ethnic group were collected for all the participants using a questionnaire. The patients were screened and diagnosed on the basis of mammography, ultrasound, followed by fine needle aspiration and tru-cut core biopsy. The specimen was collected in Formalin in a container labeled with patient name and surgical number. The equipment used was Toshiba mammography machine and histologic computed tomography machine with contrast enhanced tomography facilities. Women having age more than 40 were screened using mammography test. Mammography cannot detect tumor in dense breast tissue, while younger women are more likely to have dense breast tissue, therefor for those individuals, ultrasound was recommended. For those with BIRADS-IV and BIRADS-V lesions biopsies were undertaken, whereas for those with BIRADS-III lesions, the clinician recommended those patients with a family history for biopsy. A cut off of BIRAD-IV or above was considered as positive case of breast cancer, which was confirmed by histopathology. Ultrasound guided biopsy was done for diagnostic purposes using stereotactic biopsy facilities.

For genetic study, first one hundred cases with breast cancer were selected irrespective of their age, stage of cancer or ethnic group from a consecutive series of 302 patients with a first diagnosis of breast. Peripheral blood samples of the patients were collected in 5mL EDTA coated tubes and stored at $4^{\circ} \mathrm{C}$ before further use. All study participants signed informed written consent.

\section{Inclusion criteria:}

1. Women with early onset breast cancer with or without family history of breast cancer.

2. Women with breast cancer having family history of breast or ovarian cancer irrespective of age at diagnosis.

Extraction of DNA: Genomic DNA was extracted from $5 \mathrm{ml}$ of the blood samples using genomic DNA extraction kit (PureLink $^{\text {TM }}$ Genomic DNA Mini Kit, Thermo Scientific USA) according to manufacturer's instruction. Following extraction, the quantification of the genomic DNA was carried out by measuring the optical density at $260 \mathrm{~nm}$ using spectrophotometer. The samples were diluted to 40 $\mathrm{ng} / \mu \mathrm{L}$ to $50 \mathrm{ng} / \mu \mathrm{L}$ for further use.

Genetic Investigation: DNA samples were screened for mutations in the coding regions of $B R C A 1$ using PCR amplification followed by Sanger sequencing. 
The pairs of oligonucleotide primers were designed from intronic sequences of the gene using Primer3 software (bioinfo.ut.ee/primer3-0.4.0) (Supplementary Table-I).

The PCR reaction volume was $25 \mu \mathrm{L}$ containing 40 ng genomic DNA, 20 pmol of each primer, 200 mmol of each deoxyribonucleotide triphosphate, $1 \mathrm{U}$ of Taq DNA polymerase and $2.5 \mu \mathrm{L}$ of the buffer. All the chemicals were procured from Fermentas Inc (York, UK). The amplification conditions used were the same as described previously by Ullah et al. ${ }^{11}$ The amplified products were resolved on $2 \%$ agarose gel, stained with ethidium bromide and viewed using transilluminator. The products were purified and sequenced using Big Dye terminator cycle sequencing kit v.3.1 (Applied Biosystems, USA). The samples were aligned and analyzed for sequence variation using software BIOEDIT (version 6.0.7). Pathogenicity score for the identified variant was calculated using mutation Taster (http:/ / www.mutationtaster.org/).

\section{RESULTS}

General Characteristics of Patients: In total, 5000 women were screened at Federal Breast Cancer Screening Centre, Pakistan Institute of Medical Sciences, Islamabad during the period of three years (October 2015 to 2018). Out of which 302 women were diagnosed with breast cancer. Cancer was diagnosed base upon radiological and histopathology findings. A total of one hundred patients, all females, diagnosed with breast cancer were recruited in the study irrespective of their age or stage at the time of diagnosis. Analysis of the general characteristics showed that the study group was dominated by patients of Punjabi origin, which was further dominated by patients from Islamabad Capital Territory and Upper Potohar region of the country (Table-I). It was followed by patients of Kashmiri and Pashtun origin. The median age of the patients was 46 years with majority between 36 and 45 years of age. As shown in the Fig.1, 98\% of the cases were married, of which $63 \%$ were consanguineous marriages. The average parity was four with positive breast feeding history among

Table-I: A table showing demographics of studied population.

\begin{tabular}{lc}
\hline Ethnic Group & Percentage \\
\hline Punjabi & $73 \%$ \\
Pashtuns & $15 \%$ \\
Kashmiries & $6 \%$ \\
Others (Balochi, Sindhi, Muhajir) & $6 \%$ \\
\hline
\end{tabular}

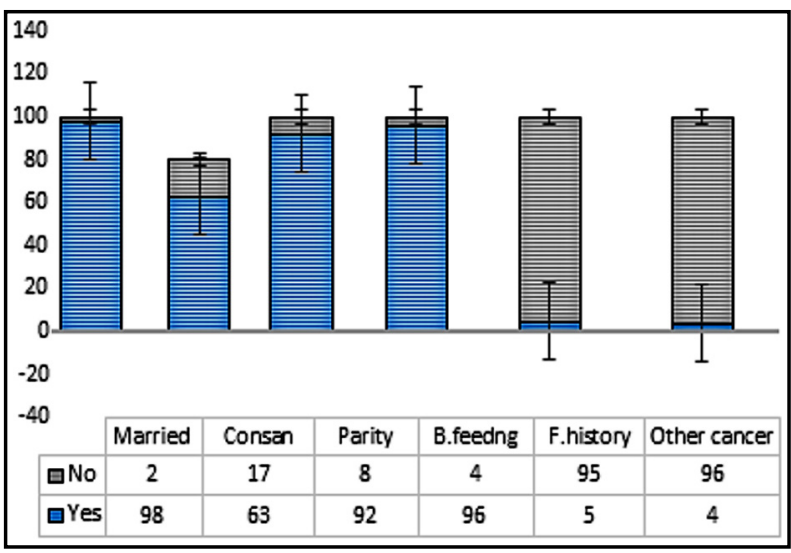

Fig.1: General characteristics of the breast cancer patients recruited for screening BRCA1 for disease causing variants.

Abbreviations: Consan= consanguinity; B.feeding= Breast feeding; F.history= Family history.

$96 \%$ of the cases. Family history of breast cancer was noted among only $5 \%$ while other cancer type was positive among $4 \%$ of the cases (Fig.1).

Genetic Investigation: Blood samples collected from 100 diagnosed breast cancer patients were screened for mutations assuming that these alterations could play a significant role in the pathogenesis of breast cancer. In total, two frame shift (p.Gly312Trpfs*8, p.Ala322Glyfs*4), six missense (p.Glu362Lys, p.Lys651Arg, p.Asp693Asn, p.Pro871Leu, p.Glu1134Lys, p.Lys1183Arg), four synonymous (p.Thr327Thr, p.Ser694Ser, p.His771His, p.Gln1135Gln), and two intronic variants (g.75407T>C, g.75401_75401delT) were detected in the BRCA1 among 79 of the total 100 patients (summarized in Table-II).

Table-II: List of mutations found in the gene BRCA1 in the present study.

\begin{tabular}{lccc}
\hline $\begin{array}{l}\text { Type of } \\
\text { mutation }\end{array}$ & $\begin{array}{c}\text { Nucleotide } \\
\text { change }\end{array}$ & Protein & $\begin{array}{c}\text { Recurrent/ } \\
\text { Novel }\end{array}$ \\
\hline Frameshift & c.932_933insC & Gly312Trpfs*8 & Novel \\
Frameshift & c.964_965insG & Ala322Glyfs*4 & Novel \\
Missense & c.1084G>A & Glu362Lys & Novel \\
Missense & c.1952A >G & Lys651Arg & Novel \\
Missense & c.2077G>A & Asp693Asn & Recurrent \\
Missense & c.2612C>T & Pro871Leu & Recurrent \\
Missense & c.3400G>A & Glu1134Lys & Novel \\
Missense & c.3548A $>$ G & Lys1183Arg & Recurrent \\
Synonymous & c.981A $>$ G & p.Thr327Thr & Novel \\
Synonymous & c.2082C>T & p.Ser694Ser & Novel \\
Synonymous & C.2311T>C & p.His771His & Novel \\
Synonymous & c.3405G $>$ A & p.Gln1135Gln & Novel \\
Intron Variant & g.75407T $>$ C & & Novel \\
Intron Variant & g.75401_75401delT & Novel \\
\hline
\end{tabular}


Following mutational analysis, clinicalpathological features were analyzed in samples with or without $B R C A 1$ alterations. Results showed that women found positive for BRCA1 mutations were younger (36 years) and expressed higher rate of lymph node involvement as compared to women without alterations. Similarly, BRCA1 group was dominated by cases expressing bilaterally affected breast tissues with lower expression of estrogen $(\mathrm{ER})$ and progesterone $(\mathrm{PgR})$ receptors as compared to non-BRCA1 cases.

\section{DISCUSSION}

All the patients recruited in the study were selected at Federal Breast Cancer Screening Centre. This dedicated breast cancer screening facility is situated at the heart of the Federal Capital and is visited by the patients from all provinces of Pakistan. Thus the data collected from patients visiting the center could help to delineate the mutational spectrum of the population of the region and contribute towards designing improvised awareness and control strategies. The data analysis showed that breast cancer patients presenting $B R C A 1$ mutations were mostly younger ( $\leq 45$ years of age), married, and had positive history of breast feeding and child bearing. The median age at the time of diagnosis was found to be 48.9 years.

Four different types of mutations were detected in DNA extracted from multiple unrelated patients (Table-II). These included frameshift, missense, synonymous and intron variations. The two frameshift variants (p.Gly312Trpfs*8, p.Ala322Glyfs*4) are predicted to result in loss of function of the BRCA1 protein either through nonsense-mediated mRNA decay or resulting in production of truncated protein, subsequently affecting DNA-repair mechanism. Three of six missense mutations (p.Asp693Asn, p.Pro871Leu, p.Lys1183Arg) have been described previously in other populations ${ }^{12-14}$ but not in Pakistani population. Three other missense variants (p.Glu362Lys, p.Lys651Arg, p.Glu1134Lys) were novel and not reported previously in any population. These three variants are located between the nuclear localization signals and the C-terminus of BRCA1 which is involved in the interaction of BRCA1 with a number of proteins that function in the DNA repair process and the cell cycle checkpoint control. ${ }^{15}$ The two intronic variants $\quad$ (g.75407T $>C, \quad$ g.75401_75401delT) identified in present study are predicted to affect the gene expression. It is highly likely that these nucleotides in the intronic regions are part of regulatory sequences of the gene affecting its expression level.

Comparison of clinical pathological features observed among women with and without alterations in the BRCA1 revealed the former were found to have high cyto-histological tumor grade, showed nodal involvement and expressed low levels of progesterone and estrogen receptors. These findings are consistent with studies previously reported from Pakistan and other parts of the world. Rashid et al. ${ }^{16}$ have reported that Pakistani women are usually diagnosed with breast cancer below 40 years of age, often present high tumor grade and show poor rate of lymph node involvement. Similarly, Leide and colleagues reported median age of diagnosis as 41 years. ${ }^{17}$ A hospital based study on breast cancer patients from Apulia, Italy supports our findings as it reported ductal invasive type, higher cytohistological tumor grade, negative ER and $\mathrm{PgR}$ status in the BRCA1 associated carcinomas. ${ }^{14}$ A possibility cannot be excluded that patients found negative for variants in the BRCA1 have variations in the $B R C A 2$.

Pakistan is a country that has faced massive migration of population and is hence composed of varying ethnic groups. ${ }^{18}$ The findings of the present study present varying mutational spectrum across different ethnic groups. Although, the study recruited relatively small number of patients and presents limited data in terms of age, ethnic group, etc., nevertheless it contributes to the studies conducted earlier by adding up to the different mutational changes described for the Pakistani population so far. This study although provides an insight into mutational spectrum of the patients in the region nevertheless it offers several limitations. The moderate sample size limited the statistical analysis. Henceforth, further population based case control studies with relatively larger sample size are suggested.

\section{CONCLUSION}

Genetic factors in the form of BRCA1 mutations are significant contributors to the high prevalence of breast cancer among Pakistani population. Therefore genetic testing for BRCA1 mutations along with $B R C A 2$ is highly crucial in order to streamline early detection and hence reduce mortality of breast cancer. 
Acknowledgments: The authors are thankful to Pakistan Science Foundation (project \#PSF/Res/CQU/Med (516)) and Higher Education Commission (project \# 1659 and 2435)" for funding the study.

\section{Conflict of Interest: None.}

\section{REFERENCES}

1. Sarwar MR, Saqib A. Cancer prevalence, incidence and mortality rates in Pakistan in 2012. Cogent Med. 2017;4:1288773.

2. Pasche B. Recent advances in breast cancer genetics. Cancer Treat Res. 2008;141:1-10.

3. Ewald IP, Ribeiro PL, Palmero EI, Cossio SL, Giugliani R, Ashton-Prolla P. Genomic rearrangements in BRCA1 and BRCA2: A literature review. Genet Mol Biol. 2009;32:437446. doi: 10.1590/S1415-47572009005000049.

4. Albertsen H, Plaetke R, Ballard L, Fujimoto E, Connolly J, Lawrence E. Genetic mapping of the BRCA1 region on chromosome 17q21. Am J Hum Genet. 1994;54:516-525.

5. Yarden RI, Pardo-Reoyo S, Sgagias M, Cowan K H, Brody $\mathrm{LC}$ et al. BRCA1 regulates the G2/M checkpoint by activating Chk1 kinase upon DNA damage. Nature Genet. 2002;30:285-289. doi: 10.1038/ng837.

6. Moatter T, Aban M, Khan S, Azam I, Pervez S. BRCA1 status in Pakistani breast cancer patients with moderate family history. J Coll Physicians Surg Pak. 2011;21:680-684. doi: 11.2011/JCPSP.680684.

7. Johannesdottir G, Gudmundsson J, Bergthorsson JT Arason A, Agnarsson BA, Eiriksdottir G et al. High prevalence of the 999del5 mutation in Icelandic breast and ovarian cancer patients. Cancer Res. 1996;56:3663-3665.

8. Szabo CI, King MC. Population genetics of BRCA1 and BRCA2. Am J Hum Genet. 1997;60:1013-1020.

9. Majeed AI. The role of the community and its impact on breast cancer awareness. Ann Pak Ins Med Sci. 2017;13:220-221.

10. Rashid MU, Zaidi A, Torres D, Sultan F, Benner A, Naqvi $B$ et al. Prevalence of BRCA1 and BRCA2 mutations in Pakistani breast and ovarian cancer patients. Int J Cancer. 2006;119:2832-2839. doi: 10.1002/ijc.22269.

11. Ullah A, Raza SI, Ali RH, Naveed AK, Jan A, Rizvi SD et al. A novel deletion mutation in the DSG4 gene underlies autosomal recessive hypotrichosis with variable phenotype in two unrelated consanguineous families. Clin Exp Dermatol. 2015;40:78-84. doi: 10.1111/ced.12457.
12. Bhatti $\mathrm{P}$, Struewing JP, Alexander $\mathrm{BH}$, Hauptmann M, Bowen L, Mateus-Pereira LH et al. Polymorphisms in DNA repair genes, ionizing radiation exposure and risk of breast cancer in U.S. Radiologic technologists. Int J Cancer. 2008;122:177-182. doi:10.1002/ijc.23066.

13. Tommasi S, Pilato B, Pinto R, Monaco A, Bruno M, Campana $\mathrm{M}$ et al. Molecular and in silico analysis of BRCA1 and BRCA2 variants. Mutat Res. 2008;644:64-70. doi: 10.1016/j. mrfmmm.2008.07.005.

14. Zhou X, Han S, Wang S, Chen X, Dong J, Shi X et al. Polymorphisms in HPV E6/E7 protein interacted genes and risk of cervical cancer in Chinese women: a case-control analysis. Gynecol Oncol. 2009;114:327-331. doi: 10.1016/j. ygyno.2009.05.011.

15. Christou CM, Kyriacou K. BRCA1 and its network of interacting partners. Biol (Basel). 2013;2:40-63. doi: 10.3390/ biology2010040

16. Rashid MU, Muhammad N, Bajwa S, Faisal S, Tahseen $\mathrm{M}$, Bermejo JL et al. High prevalence and predominance of BRCA1 germline mutations in Pakistani triple-negative breast cancer patients. BMC Cancer 2016; 16:673. doi: 10.1186/s12885-016-2698-y.

17. Liede A, Malik IA, Aziz Z, Rios Pd Pde L, Kwan E, Narod SA. Contribution of BRCA1 and BRCA2 mutations to breast and ovarian cancer in Pakistan. Am J Hum Genet 2002;71:595-606. doi:10.1086/342506

18. Umair M, Ahmad F, Ullah A. Whole Exome Sequencing as a Diagnostic Tool for Genetic Disorders in Pakistan. Pak J Med Res. 2018;57:90-91.

\section{Author's Contributions:}

AIM, AU recruited samples and collected/compiled clinical information with help of MJ, AIM, AU, MJ, performed genetic studies, and analyzed data alongside WA, Manuscript writing and revision. WA, SR. Study supervision and coordination. All the authors critically revised the manuscript and contributed to the discussion. The final version of the paper was read and approved by all authors. 